\title{
QUANTUM CRYSTALS IN NEUTRON STARS
}

\author{
V. CANUTO* and S. M. CHITRE** \\ Institute for Space Studies, Goddard Space Flight Center, NASA, New York, N.Y., U.S.A.
}

\begin{abstract}
Using the many-body techniques appropriate for quantum crystals it is shown that the deep interior of a neutron star is most likely an orderly arrangement of neutrons, protons and hyperons forming a solid. It is shown that a liquid or gas arrangement would produce higher energy. If so, a neutron star can be viewed as two solids (crust and core) permeated by a layer of ordinary or (perhaps) superfluid liquid. Astronomical evidence is in favor of such a structure: the sudden jumps in the periods of the Crab and Vela pulsars that differ by a factor of $\sim 10^{2}$ can be easily explained by the star-quake model. If the Crab is less massive than Vela (i.e., if it is not dense enough to have a solid core), the star-quakes take place in the crust whereas for Vela they occur in the core.
\end{abstract}

\section{Introduction}

It is not an exaggeration to say that the equation of state of matter at densities much beyond nuclear density is not adequately understood. An approach adopted by Banerjee et al. (1970), following a suggestion by Bethe, was to assume that when the nuclear forces become sufficiently repulsive, a possible minimum energy state could be achieved by keeping the nucleons as far away from one another as possible, i.e., by localizing them at lattice sites. This was a preliminary attempt to explore the viability of neutron crystallization and the lattice calculation was done in the harmonic approximation employing the classical Debye model. Shortly afterwards Pandharipande (1971) calculated the binding energy of dense neutron gas up to a density of the order of $7 \times 10^{15} \mathrm{~g} \mathrm{~cm}^{-3}$. He used the Reid soft-core potential in the framework of the lowest order variational approach by expanding the trial wave function as a product of single particle wave functions and the short-range correlation $a$ la Jastrow. His calculations yielded energies which were lower approximately by a factor of 1.5-2 compared to the energy of Banerjee et al. (1970) over the density range $7.5 \times 10^{14} \leq \varrho \leq 6 \times 10^{15} \mathrm{~g} \mathrm{~cm}^{-3}$. This clearly demonstrated a need to undertake a quantum mechanical treatment for the neutron lattice. Such a treatment is expected to lower the energy compared to the classical calculation by spreading the wave function around each lattice site. Moreover, the classical harmonic oscillator treatment is not adequate for a satisfactory description of a lattice in which the zero-point kinetic energy of oscillating particles becomes comparable with their potential energies. We must, therefore, work in the framework of a quantum mechanical formulation by including the effects of short-range correlation.

Recently Anderson and Palmer (1971) and Clark and Chao (1972) adopted an empirical approach based on de Boer's quantum mechanical law of corresponding states in order to examine the possibility of crystallization of neutron matter. In

* Also with the Dept. of Physics, City College of New York, N.Y.C.

** NAS-NRC Senior Research Associate. On leave of absence from the Tata Institute of Fundamental Research, Bombay. 
this approach the solidification pressure of neutron matter is estimated by scaling the known low temperature properties of quantum solids like ${ }^{3} \mathrm{He}$ to those characteristic of nucleons. Both these computations yielded a solidification pressure of the order of $5 \times 10^{27}$ atmospheres and the corresponding density for the onset of solidification of neutron matter in the vicinity of $5 \times 10^{14} \mathrm{~g} \mathrm{~cm}^{-3}$. Quite apart from the fact that the nucleon-nucleon forces are spin, state, and isospin dependent, which can scarcely be embodied in a single effective potential, it is not altogether justified simply to scale the potential. Such a scaling, even though it may give an over-all agreement between the well-depth and the core-radius of various potentials, almost certainly cannot produce a good fit to the tail of the potential where it remains a difficult task to match the derivatives of the potential - features which, in fact, are critical from the point of view of the stability of the lattice. We have displayed in Figure 1 the singlet ${ }^{1} S_{0}$ nucleon-nucleon potential along with the appropriately scaled Lennard-Jones potential from which it can be readily seen that even though the core radius and the depth can be fitted to a desired accuracy, the quantitative difference in the relative displace-

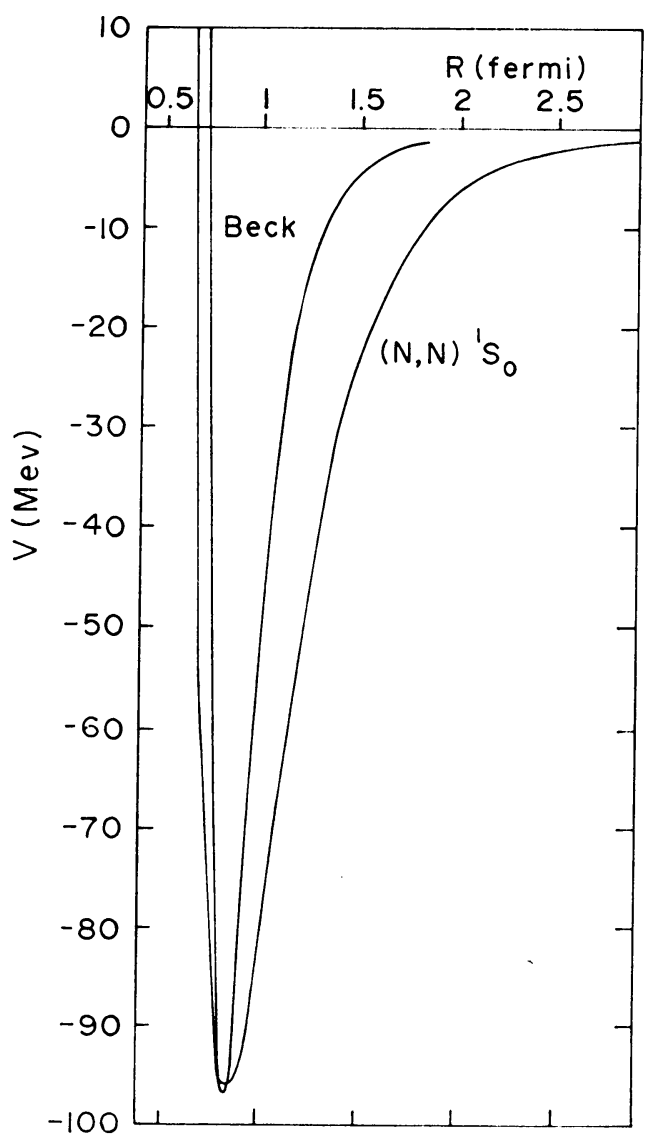

Fig. 1. Singlet ${ }^{1} S_{0}$ Reid potential and scaled L-J potential. 


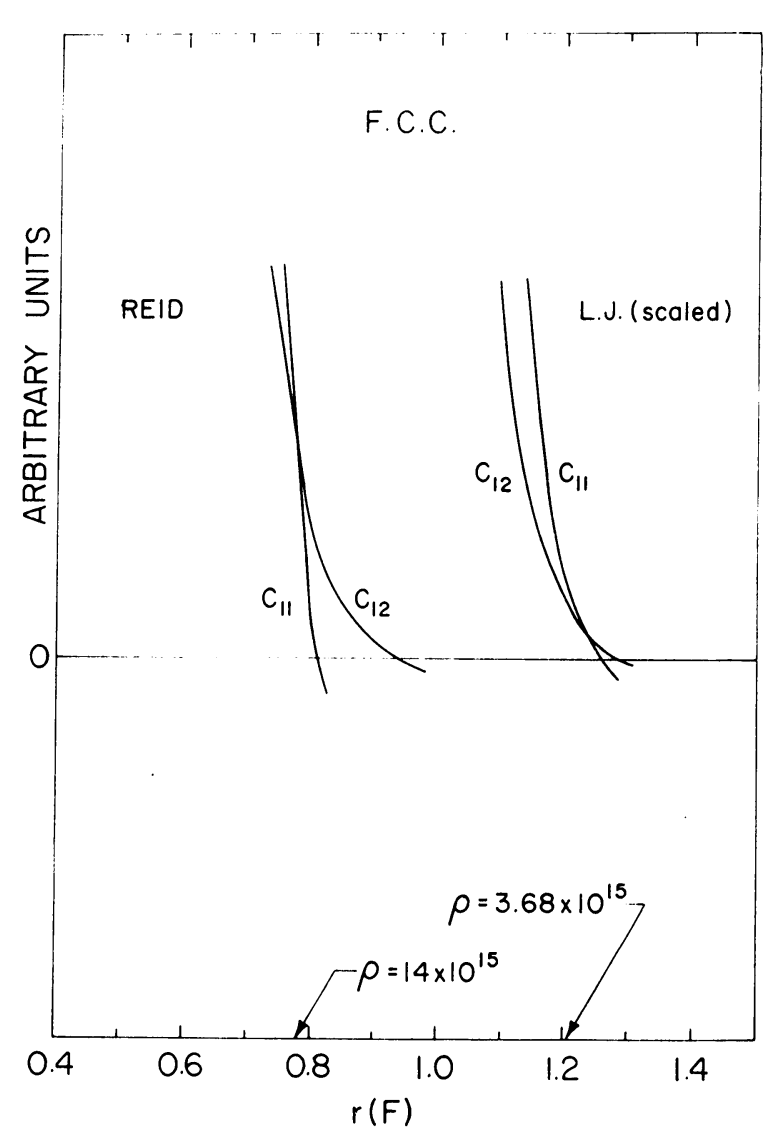

Fig. 2. Elastic constants $C_{11}, C_{12}$ computed for an FCC lattice using Reid and L-J (scaled).

ment of the points of inflection and different slopes of the two potentials are quite noteworthy. The elastic constants computed by using the nuclear and scaled potentials are shown in Figure 2 where the interesting feature is the point at which the elastic constants go soft. On the scaled $\mathrm{LJ}$ potential the elastic constants indicate an onset of the solid phase at a density $\sim 3.7 \times 10^{15} \mathrm{~g} \mathrm{~cm}^{-3}$, while the elastic constants computed using the Reid soft-core potential do not permit the solid to exist until a density approximately four times higher is reached. We feel that simply estimating the solidification pressure does not by any means provide the complete answer, rather we must test a given lattice structure for stability to determine whether the solid state is permissible on mechanical grounds.

\section{Many-Body Treatment}

The many-body treatment which we have used is an extension of the $T$-matrix approach to quantum crystals recently discussed by Brandow (1972), and an excellent review can be found in a paper by Guyer (1969). The Slater determinant for a system of $N$ 
particles is built up by single-particle wave functions which we take to be of Gaussian form:

$$
\phi(r)=\alpha^{3 / 2} \pi^{-3 / 4} e^{-\alpha^{2} / 2|r-R|^{2}},
$$

where $R$ is the coordinate of the lattice site around which the particle is supposed to perform an oscillatory motion. Clearly (1) is the eigenfunction of an harmonic oscillator potential $U(r)$, centered around the lattice site $R$, i.e., $U(r)=1 / 2 m \omega^{2}|r-R|^{2}$, where the frequency $\omega$ enters the wave function through the parameter $\alpha^{-1}=(m \omega / \hbar)^{-1 / 2}$ which represents the spread of $\phi$ around the lattice site. For determining $\alpha$ we do a Hartree calculation by taking the single particle potential to be given by

$$
U\left(r_{1}\right)=\sum_{j} \phi_{j}^{*}\left(r_{2}\right) V\left(r_{1}-r_{2}\right) \phi_{j}\left(r_{2}\right) \mathrm{d}^{3} r_{2}
$$

where the two-body nucleon-nucleon potential is taken to be Reid's phenomenological soft-core potential. The index $j$ runs over successive neighbors (shells) from a given particle located at $R_{1}$. The values of $\alpha$ obtained by solving (2) are then used to start the full HF equations which finally give the form of $\phi$.

Among the various degrees of sophistication that one can use to write the equation for the correlated or perturbed wave function $\psi$ we shall employ the one first employed by Guyer and Zane (1969) where $\psi$ is assumed to satisfy an equation analogous to the Bethe-Goldstone equation of motion, i.e.,

$$
[T(1)+T(2)+U(1)+U(2)+V(12)] \psi=\varepsilon \psi .
$$

Here $T(1)$ and $T(2)$ are respectively the kinetic energies, $U(1)$ and $U(2)$ are the harmonoscillator potentials of particles 1 and 2 , and $V(12)$ is the two-body interaction potential. Equation (4) is basically the equation of motion of two particles, each moving in a harmonic oscillator potential centered around two different lattice sites and in addition, interacting through a two-body potential. If one uses for $U(1)$ and $U(2)$ the form given by (2), then (4) becomes

Here

$$
\left[T_{R}+T_{r}+\frac{1}{4} m \omega^{2}(r-\Delta)^{2}+m \omega^{2}(R-\delta)^{2}+V(r)\right] \psi=\varepsilon \psi
$$

$$
\begin{aligned}
& T_{R}=-\frac{\hbar^{2}}{4 m} \nabla_{R}^{2}, \quad T_{r}=-\frac{\hbar^{2}}{m} \nabla_{r}^{2}, \quad r_{1}-r_{2}=r, \quad r_{1}+r_{2}=2 R, \\
& R_{1}-R_{2}=\Delta, \quad R_{1}+R_{2}=2 \delta .
\end{aligned}
$$

Since the nuclear interaction potential is highly angular momentum dependent, we write the full angular momentum decomposition of $\psi$ in the form:

$$
\psi(\mathbf{r})=\sum_{l=0}^{\infty} \sum_{J}(2 l+1) i^{l} \sqrt{\frac{4 \pi}{2 l+1}}\left(l 0 S M_{\mathrm{s}} \mid J M_{\mathrm{s}}\right) \sum_{l^{\prime}} \psi_{l l^{\prime}}^{J S}(r) Y_{l^{\prime} J S}^{M_{\mathrm{s} S}}(\Omega)
$$

in the usual notation. The second summation over $l^{\prime}$ is introduced to take into account the presence of tensor forces. Equation (5) contains a $\cos \theta$ term which, much like the Stark effect, couples $l$ with $l \pm 1$, but of course does not couple spins and therefore 
it is to be expected that the singlet states ${ }^{1} S_{0},{ }^{1} P_{1},{ }^{1} D_{2}$ will be coupled by such a term and analogously the triplet states ${ }^{3} S_{1},{ }^{3} \tilde{S}_{1},{ }^{3} P_{0},{ }^{3} P_{1},{ }^{3} P_{2},{ }^{3} D_{1},{ }^{3} \tilde{D}_{1},{ }^{3} D_{2}$ will be linked together. After inserting (6) into (5) and with a certain amount of algebraic manipulation, we get $\left(x=r / r_{0}, d=\Delta / r_{0}, a=\alpha r_{0}\right)$ :

$$
\begin{cases}\text { SINGLET } & \\ h_{0}^{\prime \prime}+\left(E-U_{0}\right) h_{0}+a(x, d) h_{1} & =0 \\ h_{1}^{\prime \prime}+\left(E-U_{1}\right) h_{1}+\frac{1}{3} a(x, d)\left[h_{0}-2 h_{2}\right] & =0 \\ h_{2}^{\prime \prime}+\left(E-U_{2}\right) h_{2}-\frac{2}{5} a(x, d) h_{1} & =0\end{cases}
$$

where the following notation has been used

$$
\begin{array}{ll}
{ }^{1} S_{0}: & r \psi_{00}^{00}=h_{0}, \quad a(x, d)=\frac{1}{2} a^{4} x d \\
{ }^{1} P_{1}: & r \psi_{11}^{10}=h_{1} \\
{ }^{1} D_{2}: & r \psi_{22}^{20}=h_{2}
\end{array}
$$

\section{TRIPLET}

$$
\begin{aligned}
& h_{3}^{\prime \prime}+\left(E-U_{3}\right) h_{3}-\sqrt{8} V_{T} \frac{2 \mu r_{0}^{2}}{h^{2}} \tilde{h}_{3}+\frac{1}{9} a(x, d)\left[h_{4}+3 h_{5}+5 h_{6}\right]=0 \\
& \tilde{h}_{3}^{\prime \prime}+\left(E-\tilde{U}_{3}\right) \tilde{h}_{3}-\sqrt{ } 8 V_{T} \frac{2 \mu r_{0}^{2}}{\hbar^{2}} h_{3}-\frac{\sqrt{2}}{9} a(x, d)\left[h_{4}-\frac{3}{2} h_{5}+\frac{1}{2} h_{6}\right]=0 \\
& h_{4}^{\prime \prime}+\left(E-U_{4}\right) h_{4}+\quad 0 \quad+\frac{1}{3} a(x, d)\left[\left(h_{3}+\sqrt{2} \tilde{h}_{7}\right)+\right. \\
& \left.-\sqrt{2}\left(\tilde{h}_{3}+\sqrt{2} h_{7}\right)\right]=0 \\
& h_{5}^{\prime \prime}+\left(E-U_{5}\right) h_{5}+ \\
& +\frac{1}{3} a(x, d)\left[\left(h_{3}-\frac{1}{\sqrt{2}} h_{7}\right)\right. \\
& \left.+\frac{1}{\sqrt{2}}\left(\tilde{h}_{3}-\frac{1}{\sqrt{2}} h_{7}\right)-\frac{3}{2} h_{8}\right]=0 \\
& h_{6}^{\prime \prime}+\left(E-U_{6}\right) h_{6}+\quad 0 \quad+\frac{1}{3} a(x, d)\left[\left(h_{3}+\frac{1}{5 \sqrt{2}} \tilde{h}_{7}\right)+\right. \\
& \left.-\frac{1}{5 \sqrt{2}}\left(\tilde{h}_{3}+\frac{1}{5 \sqrt{2}} h_{7}\right)-\frac{3}{10} h_{8}\right]=0 \\
& h_{7}^{\prime \prime}+\left(E-U_{7}\right) h_{7}-\sqrt{8} V_{T} \frac{2 \mu r_{0}^{2}}{\hbar^{2}} \tilde{h}_{7}-\frac{1}{9} a(x, d)\left[2 h_{4}+\frac{3}{2} h_{5}+\frac{1}{10} h_{6}\right]=0 \\
& \tilde{h}_{7}^{\prime \prime}+\left(E-\tilde{U}_{7}\right) \tilde{h}_{7}-\sqrt{8} V_{T} \frac{2 \mu r_{0}^{2}}{\hbar^{2}} h_{7}+\frac{\sqrt{2}}{9} a(x, d)\left[h_{4}-\frac{3}{2} h_{5}+\frac{1}{2} h_{6}\right]=0 \\
& h_{8}^{\prime \prime}+\left(E-U_{8}\right) h_{8}-\quad 0 \quad-\frac{1}{10} a(x, d)\left[3 h_{5}+h_{6}\right] \quad=0
\end{aligned}
$$

with the following notation

$$
\begin{array}{llll}
{ }^{3} S_{1}: & r \psi_{00}^{11}=h_{3} & { }^{3} P_{2}: & r \psi_{11}^{21}=h_{6} \\
{ }^{3} \tilde{S}_{1}: & r \psi_{02}^{11}=\tilde{h}_{3} & { }^{3} D_{1}: & r \psi_{22}^{11}=h_{7}
\end{array}
$$




$$
\begin{array}{llll}
{ }^{3} P_{0}: & r \psi_{11}^{01}=h_{4} & { }^{3} \tilde{D}_{1}: & r \psi_{20}^{11}=\tilde{h}_{7} \\
{ }^{3} P_{1}: & r \psi_{11}^{11}=h_{5} & { }^{3} D_{2}: & r \psi_{22}^{21}=h_{8}
\end{array}
$$

The potentials $U_{k}$ and $\tilde{U}_{k}$ are given by

$$
\begin{aligned}
& U_{k}=\frac{1}{4} a^{4} x^{2}+\frac{2 \mu r_{0}^{2}}{\hbar^{2}} V_{k}+\frac{l(l+1)}{x^{2}} \\
& \tilde{U}_{3}=\frac{1}{4} a^{4} x^{2}+\frac{2 \mu r_{0}^{2}}{\hbar^{2}} V\left({ }^{3} D_{1}\right)+\frac{6}{x^{2}} \\
& \tilde{U}_{7}=\frac{1}{4} a^{4} x^{2}+\frac{2 \mu r_{0}^{2}}{\hbar^{2}} V\left({ }^{3} S_{1}\right) .
\end{aligned}
$$

The two-body potentials $V_{k}$ are taken from Reid (1968).

It can be seen that the two sets of equations are intrinsically coupled by the solidstate term. Only two normalization conditions are therefore necessary: one for each set of singlets and triplets. The solution of the $3+8$ differential equations presented us with considerable numerical difficulty in the absence of any Sturm-Liouville type theorems for coupled equations, and the search for the ground state energy of successive shells was a painfully laborious process. The major concern was to make certain that the energy eigenvalues being computed were actually the lowest, i.e., the eigenfunctions $h_{k}$ 's had no nodes.

The energy per particle, $E / N$, consists of the kinetic energy, which for displaced harmonic oscillator wave functions takes the form

$$
E_{\text {K.E. }}=\frac{3 \hbar^{2}}{4 m} \alpha^{2} N
$$

together with the potential energy

$$
\begin{aligned}
E_{\text {P.E. }} & =\sum_{i<j} \int \Phi(i j) V(i j) \Psi(i j) \mathrm{d}^{3} r_{i} \mathrm{~d}^{3} r_{j} \\
& =\frac{1}{2} N \sum_{\Delta} N_{\Delta} \frac{\int \Phi(r) V(r) \Psi(r) \mathrm{d}^{3} r}{\int \Phi(i j) \mathrm{d}^{3} r_{i} \mathrm{~d}^{3} r_{j}}
\end{aligned}
$$

where $\Phi(i j)$ is the two-body wave function, i.e., $\Phi=\phi\left(r_{1}\right) \phi\left(r_{2}\right)$ and $N_{\Delta}$ is the number of particles at distance $\Delta$ from the one chosen as the origin.

\section{Elastic Constants}

We must test the system of nucleons arranged in a lattice structure for stability against small deformations. There have been several attempts to derive criteria for the melting 
of a solid, the best known being Lindemann's rule. This rule gives an empirical criterion that a solid melts when the amplitude of oscillation of a particle becomes a sizable fraction of the nearest neighbor distance. Such a criterion can only be regarded as a convenient 'rule of thumb' designed to test semi-quantitatively the stability of a crystalline structure and indeed, a complete theory of melting must examine the detailed stability of a lattice when it is deformed under shearing stresses.

Any lattice has to satisfy the requirement that its energy density must have a stationary value at equilibrium. However, for the structure to be stable, the energy must have a positive-definite quadratic form, thus increasing its value while undergoing a small strain. Following the notation of Born and Huang (1950), when a cube of side $2 a$ is deformed homogeneously, the energy to the second order of deformation in terms of the strain components $e_{\alpha \beta}$ comes out to be

$$
\begin{aligned}
E(\delta)=E(0) & +\frac{1}{2} C_{11}\left(e_{x x}^{2}+e_{y y}^{2}+e_{z z}^{2}\right)+C_{12}\left(e_{y y} e_{z z}+e_{z z} e_{x x}+e_{x x} e_{y y}\right)+ \\
& +\frac{1}{2} C_{44}\left(e_{y z}^{2}+e_{z x}^{2}+e_{x y}^{2}\right),
\end{aligned}
$$

Here $E(0)$ is the energy of the undeformed cube whose lattice points can be described by $\mathbf{R}_{0}=\left(l_{1} a, l_{2} a, l_{3} a\right),\left(l_{1}, l_{2}, l_{3}\right.$ being integers $), \Phi$ is the potential, and

$$
\begin{aligned}
& C_{11}=\frac{2 a}{\gamma} \sum D^{2} \Phi l_{1}^{4}-P, \\
& C_{12}=\frac{2 a}{\gamma} \sum D^{2} \Phi l_{1}^{2} l_{2}^{2}-P, \\
& C_{44}=\frac{2 a}{\gamma} \sum D^{2} \Phi l_{1}^{2} l_{2}^{2}-P .
\end{aligned}
$$

and $\gamma=4(\mathrm{BCC}), \gamma=2(\mathrm{FCC}), V=\gamma a^{3}$, the summation extending over various shells. The quadratic form in (5) is positive definite provided

$$
\begin{aligned}
& C_{11}+2 C_{12}>0 \text { (Sublimation: lattice has no cohesion), } \\
& C_{44}>0 \text { (Melting: lattice unstable against shearing stresses), } \\
& C_{11}-C_{12}>0 \text { (Gel: elastic resistance against shearing stresses). }
\end{aligned}
$$

For a lattice structure to be stable we must demand the satisfaction of all of the foregoing conditions.

\section{Results}

We have attempted three different ordered structures: $\mathrm{BCC}(($ body-centered cubic $)$, FCC (face-centered cubic) and HCP (hexagonal close packing). A major complication arises because of the possible spin configurations: for a given solid structure, say BCC, one could in principle arrange the nucleons in a large number of ways depending upon the spin configuration. In a BCC lattice one could start with any site with a neutron with its spin up; in the next site on the same cube one can put $N_{\uparrow}, N_{\downarrow}, P_{\uparrow}$ or $P_{\downarrow}$. In the next site one has again a similar situation. We have tried several configurations with an arbitary spin arrangement just to ensure that such arrangements are not 


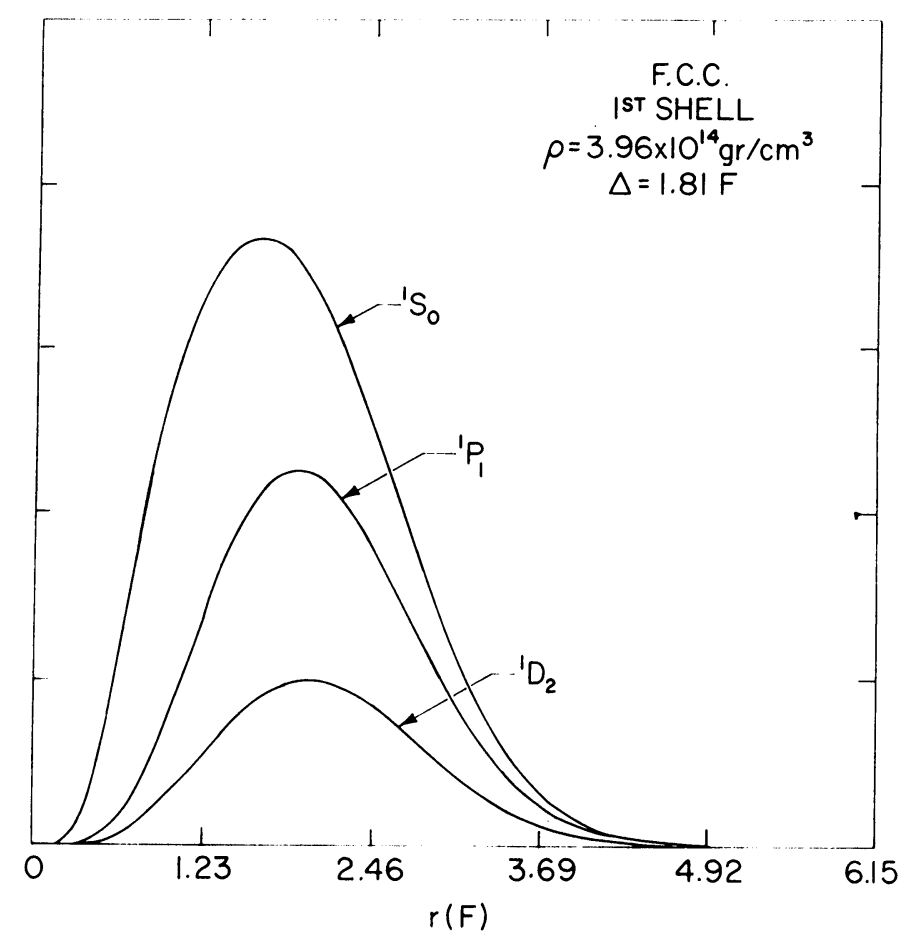

Fig. 3. Singlet state wave functions vs. $r$ at a given density.

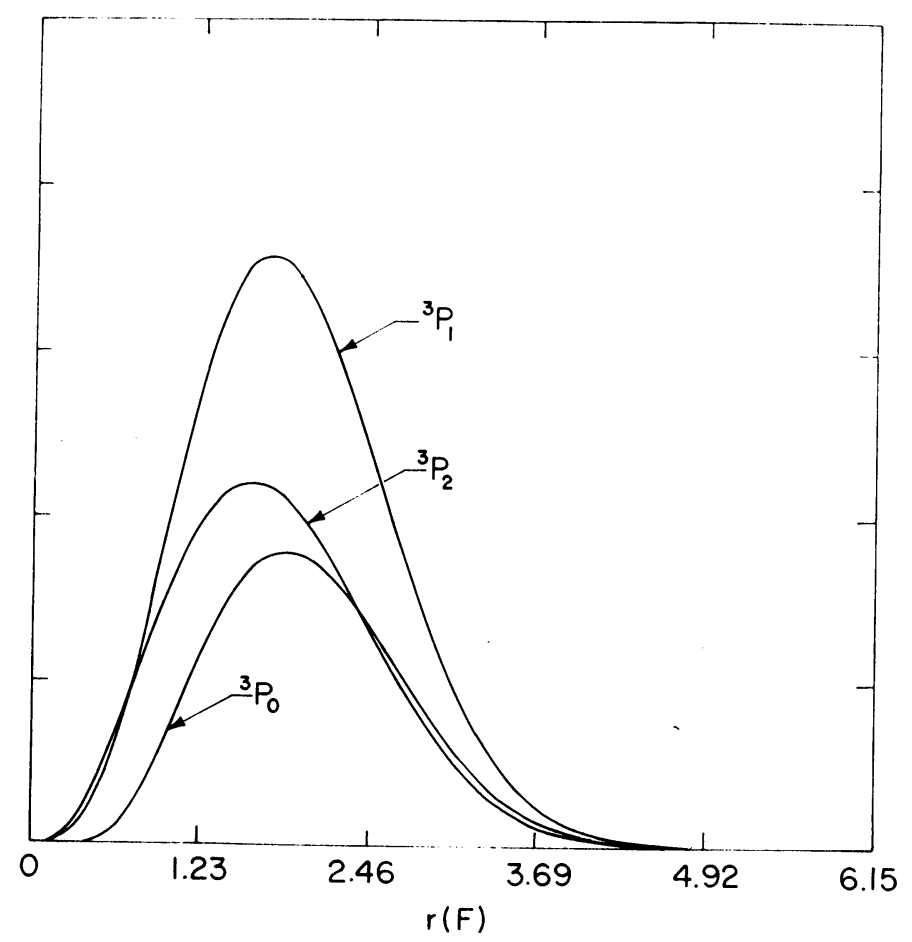

Fig. 4. Triplet state wave functions vs. $r$ at a given density. 


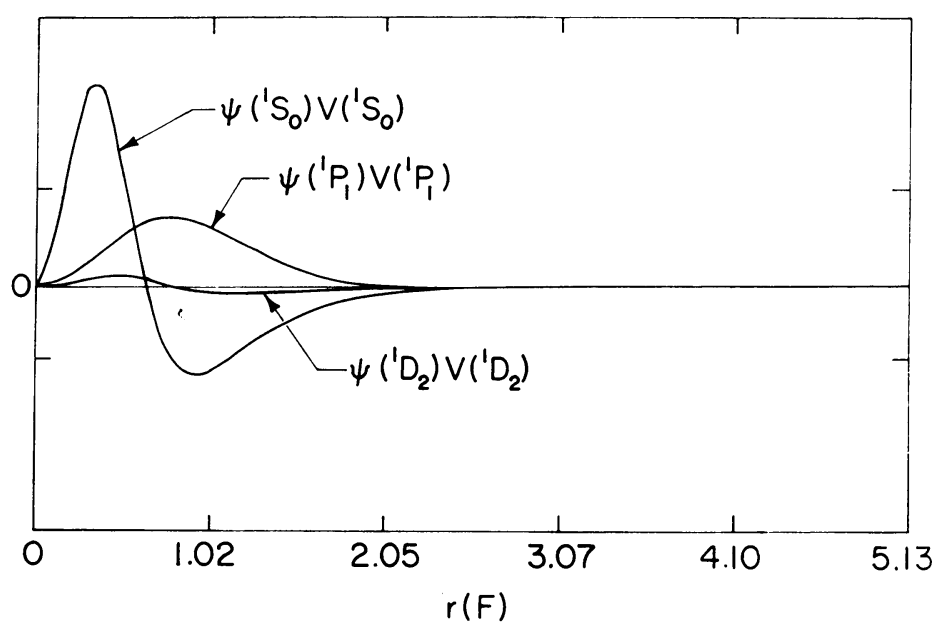

Fig. 5. The product $h V$ vs. $r$ for the singlet states.

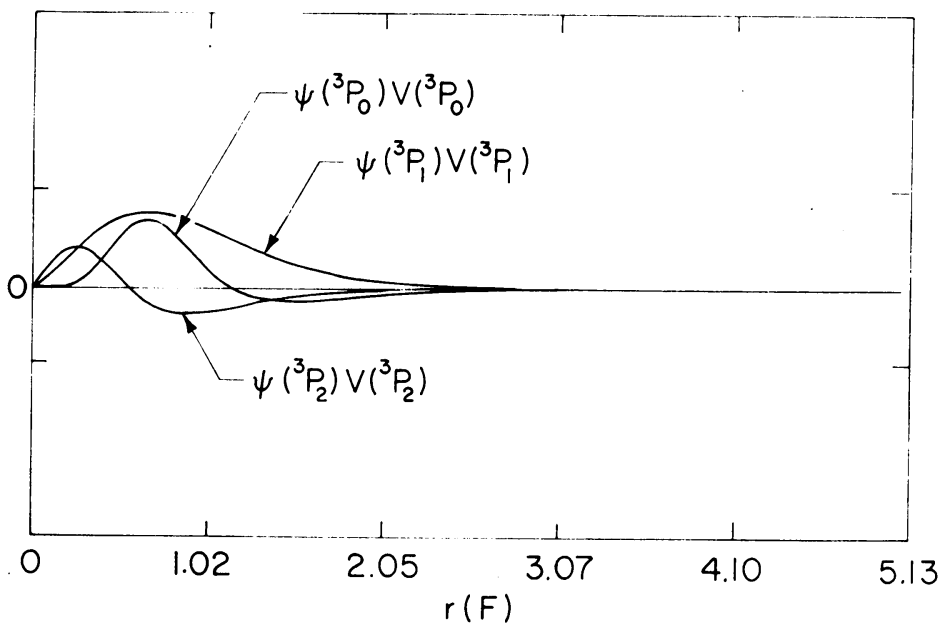

Fig. 6. The product $h V$ vs. $r$ for the triplet states.

TABLE I

Energy per particle $(\mathrm{MeV})$ vs. $10^{-15} \varrho\left(\mathrm{g} \mathrm{cm}^{-3}\right)$ for various configurations with mixed spins

\begin{tabular}{lllll}
\hline $\begin{array}{l}10^{-15} \varrho \\
\left(\mathrm{g} \mathrm{cm}^{-3}\right)\end{array}$ & $\begin{array}{l}\text { FCC } \\
\mathrm{N}: \mathrm{P}=1: 1\end{array}$ & $\begin{array}{l}\text { BCC } \\
\text { Pure neutrons }\end{array}$ & $\begin{array}{l}\text { HCP } \\
\text { Pure neutrons }\end{array}$ & $\begin{array}{l}\text { FCC } \\
\text { Pure neutrons }\end{array}$ \\
\hline & & & & \\
1.41 & 107.4 & 131.6 & 118.4 & 114.5 \\
1.832 & 181.2 & 188.7 & 174.8 & 171.8 \\
2.596 & 355.9 & 310.3 & 293.7 & 282.5 \\
3.344 & 548.9 & 472.5 & 441.6 & 428.5 \\
4.398 & 911.3 & 720.5 & 684.1 & 652.4 \\
5.237 & 1194.0 & 923.2 & 884.7 & 864.6
\end{tabular}


TABLE II

FCC pure neutrons mixed spins

\begin{tabular}{|c|c|c|c|c|c|c|}
\hline $\begin{array}{l}10^{-15} Q \\
\left(\mathrm{~g} \mathrm{~cm}^{-3}\right)\end{array}$ & $\begin{array}{l}\Delta \\
\text { (fermi }\end{array}$ & $\begin{array}{l}\alpha^{-1}(\mathrm{H} . \mathrm{F} .) \\
\text { (fermi) }\end{array}$ & $\begin{array}{l}\text { K.E. } \\
(\mathrm{MeV})\end{array}$ & $\begin{array}{l}\text { P.E. } \\
(\mathrm{MeV})\end{array}$ & $\begin{array}{l}B / N \\
(\mathrm{MeV})\end{array}$ & \\
\hline 1.41 & 1.188 & 0.460 & 146.4 & -31.9 & 114.5 & \\
\hline 1.603 & 1.138 & 0.435 & 163.8 & -24.2 & 139.6 & 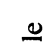 \\
\hline 1.832 & 1.089 & 0.410 & 183.9 & -12.1 & 171.8 & $\vec{\sigma}$ \\
\hline 2.294 & 1.010 & 0.376 & 219.4 & 19.6 & 239.0 & $\ddot{\tilde{\omega}}$ \\
\hline 2.596 & 0.969 & 0.361 & 238.3 & 44.2 & 282.5 & \\
\hline 3.344 & 0.891 & 0.323 & 297.2 & 131.3 & 428.5 & \\
\hline 3.776 & 0.855 & 0.310 & 322.3 & 186.9 & 509.2 & \\
\hline 4.398 & 0.813 & 0.291 & 366.0 & 286.4 & 652.4 & \\
\hline 5.237 & 0.767 & 0.271 & 421.9 & 442.7 & 864.6 & \\
\hline
\end{tabular}

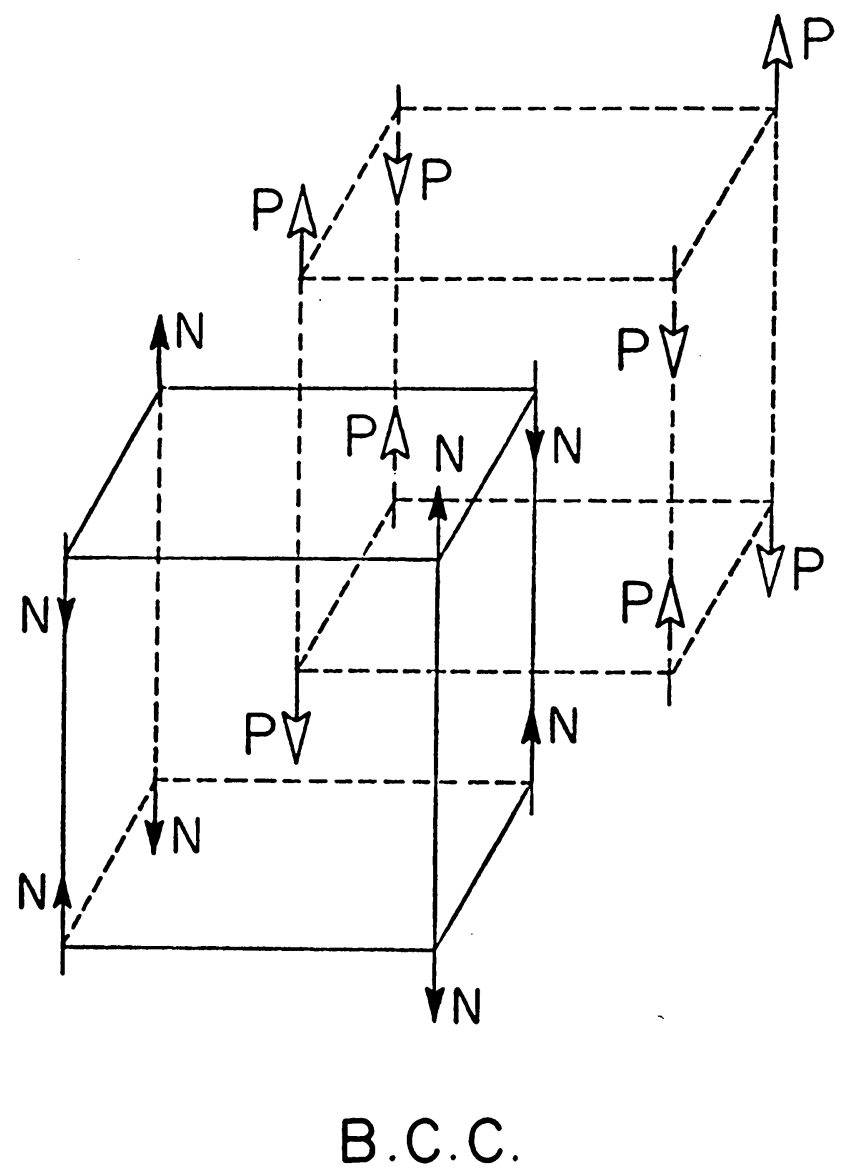

Fig. 7. BCC configuration for an equal number of neutrons and protons. 
TABLE III

FCC pure neutrons (mixed spins) elastic constants

\begin{tabular}{llll}
\hline $\begin{array}{l}10^{-15} \times Q \\
\left(\mathrm{~g} \mathrm{~cm}^{-3}\right)\end{array}$ & $\begin{array}{l}10^{-36} \times c_{11} \\
\left(\text { dyne } \mathrm{cm}^{-2}\right)\end{array}$ & $\begin{array}{l}10^{-36} \times c_{12} \\
\left(\text { dyne } \mathrm{cm}^{-2}\right)\end{array}$ & $\begin{array}{l}10^{-36} \times c_{44} \\
\left(\text { dyne } \mathrm{cm}^{-2}\right)\end{array}$ \\
\hline 5.237 & 38.46 & 14.40 & 7.55 \\
4.398 & 22.625 & 8.602 & 4.142 \\
3.776 & 14.529 & 6.052 & 3.212 \\
3.344 & 9.286 & 4.065 & 1.985 \\
2.596 & 4.164 & 1.683 & 0.675 \\
1.832 & 1.065 & 0.5414 & 0.1084 \\
1.603 & 0.5177 & 0.3386 & 0.0326 \\
1.41 & 0.1883 & 0.1838 & -0.0362 \\
\hline
\end{tabular}

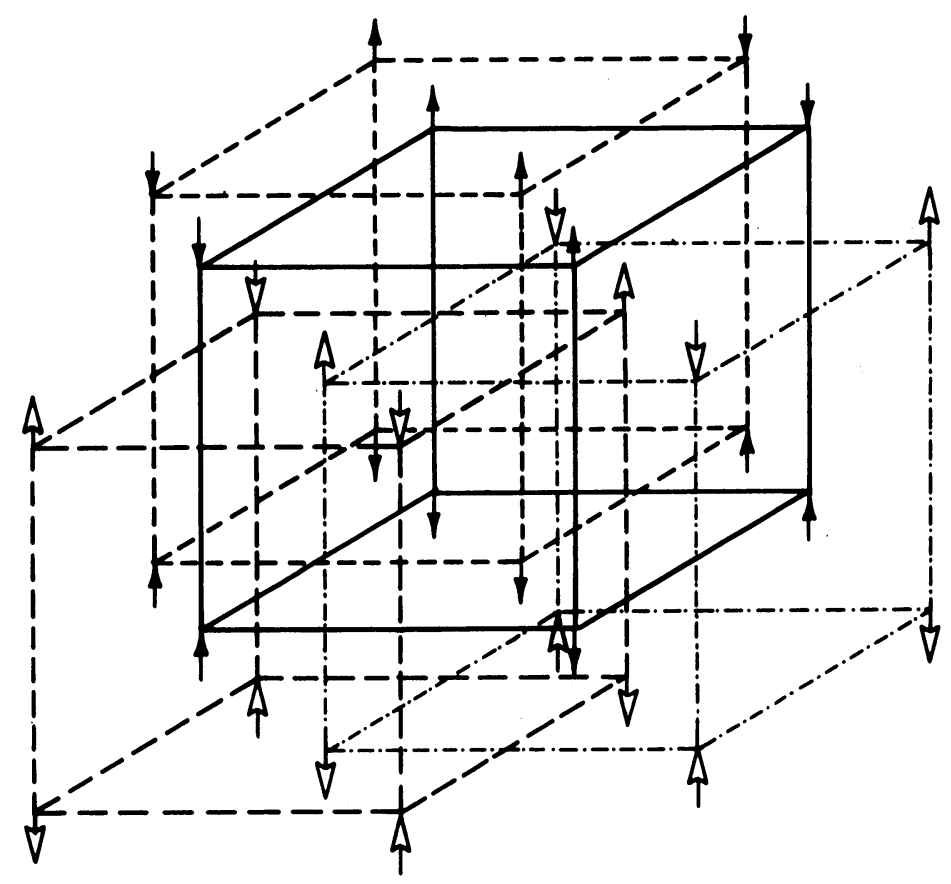

F.C.C.

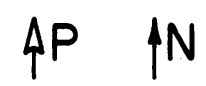

Fig. 8. FCC configuration.

energetically convenient. We were then able to establish that for a given configuration the minimum for energy was achieved whenever the spins of neighboring particles were symmetrically arranged, i.e., in the same cube the particles on neighboring sites should have anti-parallel spins (mixed spins). An analogous situation was 
found to hold for the other two configurations studied, namely FCC and HCP.

For a given configuration with a prescribed spin arrangement the system of $3+8$ equations has to be solved for each shell to yield the lowest eigenvalue. The resulting $h_{k}$ 's are inserted in the energy expression and the computations are carried far enough (approximately up to 24 shells) that contributions of further shells are unimportant. The resulting singlet wave functions ${ }^{1} S_{0},{ }^{1} P_{1},{ }^{1} D_{2}$, and the triplet wave functions ${ }^{3} P_{0},{ }^{3} P_{1},{ }^{3} P_{2}$, are respectively displayed in Figures 3 and 4 and the products $h V$ for the two cases are shown in Figures 5 and 6.

TABLE IV

FCC pure neutrons - mixed spins equation of state

\begin{tabular}{lllll}
\hline $\begin{array}{l}10^{-15} Q \\
\left(\mathrm{~g} \mathrm{~cm}^{-3}\right)\end{array}$ & $\begin{array}{l}E / N \\
(\mathrm{MeV})\end{array}$ & $\begin{array}{l}10^{-36} \times \varepsilon \\
\left(\mathrm{erg} \mathrm{cm}^{-3}\right)\end{array}$ & $\begin{array}{l}10^{-36} \times P \\
\left(\text { dyne } \mathrm{cm}^{-2}\right)\end{array}$ & $\Gamma$ \\
\hline & & & & \\
1.0 & 75.50 & 0.969 & 0.101 & 1.714 \\
1.2 & 96.88 & 1.187 & 0.150 & 2.082 \\
1.4 & 119.33 & 1.415 & 0.217 & 2.330 \\
1.6 & 143.33 & 1.654 & 0.304 & 2.479 \\
1.8 & 169.08 & 1.906 & 0.412 & 2.545 \\
2.0 & 196.08 & 2.170 & 0.541 & 2.567 \\
2.2 & 225.63 & 2.448 & 0.693 & 2.647 \\
2.4 & 256.49 & 2.741 & 0.877 & 2.799 \\
2.6 & 289.42 & 3.052 & 1.100 & 2.888 \\
2.8 & 324.54 & 3.381 & 1.355 & 2.764 \\
3.0 & 361.51 & 3.729 & 1.622 & 2.514 \\
3.2 & 399.71 & 4.095 & 1.891 & 2.326 \\
3.4 & 438.66 & 4.477 & 2.174 & 2.369 \\
3.6 & 478.48 & 4.878 & 2.505 & 2.625 \\
3.8 & 519.71 & 5.299 & 2.909 & 2.883 \\
4.0 & 562.85 & 5.743 & 3.383 & 2.985 \\
4.2 & 608.12 & 6.212 & 3.905 & 2.918 \\
4.4 & 655.31 & 6.706 & 4.449 & 2.751 \\
4.6 & 704.07 & 7.225 & 4.999 & 2.581 \\
4.8 & 754.00 & 7.769 & 5.557 & 2.489 \\
5.0 & 804.90 & 8.336 & 6.144 & 2.514 \\
\hline
\end{tabular}

The geometrical configuration of the nucleons for BCC, FCC and HCP respectively are displayed in Figures 7, 8, and 9 and Table I summarizes the energy per particle as a function of the density for the three configurations. On energetic grounds it is convenient to have only neutrons at the high density end as can be seen from a comparison of the energies computed for an FCC consisting of pure neutrons and one with an equal number of neutrons and protons. The FCC structure made up of neutrons with mixed spins does indeed appear to be the lowest energy configuration and the detailed results for such a system are presented in Table II where the density is given in the first column, the nearest neighbor distance in the second, the spread of the wave function in the third, with the kinetic, potential, and total energy per particle following in order. The region of stability is indicated on the right where it is shown 
that the lattice is stable only in the density range upwards of $\sim 1.5 \times 10^{15} \mathrm{~g} \mathrm{~cm}^{-3}$. The elastic constants $C_{11}, C_{12}, C_{44}$ are shown in Table III as a function of the density: it can be seen that $C_{44}<0$ for $\varrho \$ 1.5 \times 10^{15} \mathrm{~g} \mathrm{~cm}^{-3}$ indicating that the FCC structure made up of neutrons can withstand shearing stresses at a density upwards of this value. The spread of the wave function as measured by $\alpha^{-1}$ (fermi) vs. the density is exhibited in Figure 10 along with the nearest neighbor distance: it can be readily seen that $\alpha^{-1}$ is about a third of the nearest neighbor distance over the density range $1.41 \times 10^{15} \leq \varrho \leq 5.237 \times 10^{15} \mathrm{~g} \mathrm{~cm}^{-3}$.

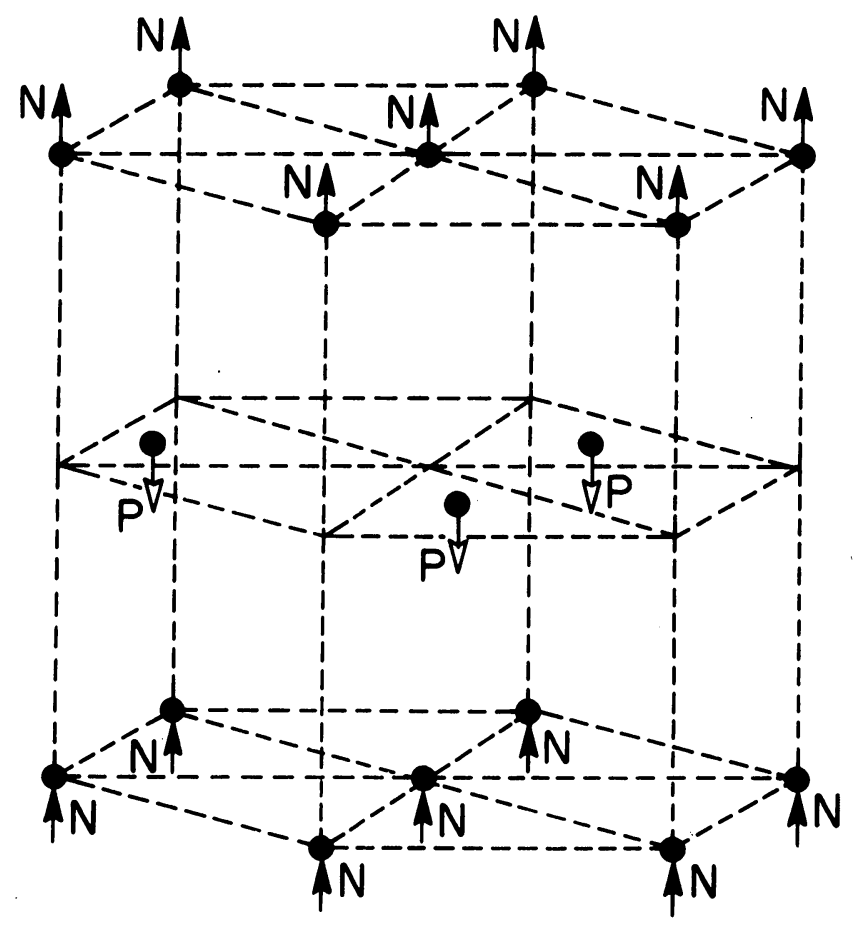

H. C.P.

Fig. 9. HCP configuration.

The equation of state derived by arranging neutrons with their spins mixed in an FCC lattice is summarized in Table IV. Here the first column lists the density, the second the energy per particle, the third the mass-energy density, the fourth the pressure derived by using the relation $P=-(\partial E / \partial V)$ and the last column shows the adiabatic index $\Gamma=(\varepsilon+P) P^{-1} \partial P / \partial \varepsilon$. Figure 11 exhibits a comparison of our energies vs. density (obtained on the assumption that nucleons are arranged in a lattice) and the energies obtained for a neutron gas by Pandharipande. It is clear that at the high density end a lattice made up of neutrons is energetically most convenient. The interes- 
ting feature to be noted is that the curves cross around $\varrho \cong 1.5 \times 10^{15} \mathrm{~g} \mathrm{~cm}^{-3}$ where the stability analysis shows that $C_{44}<0$ (i.e., the lattice cannot withstand any shearing stresses at a lower density). Below $\varrho \cong 1.5 \times 10^{15} \mathrm{~g} \mathrm{~cm}^{-3}$ the energies obtained by doing a lattice calculation exceed the corresponding energies given by a gas computation. We should therefore like to conclude that our computation seems to indicate a possible solid phase for cold matter at densities exceeding $1.5 \times 10^{15} \mathrm{~g} \mathrm{~cm}^{-3}$.

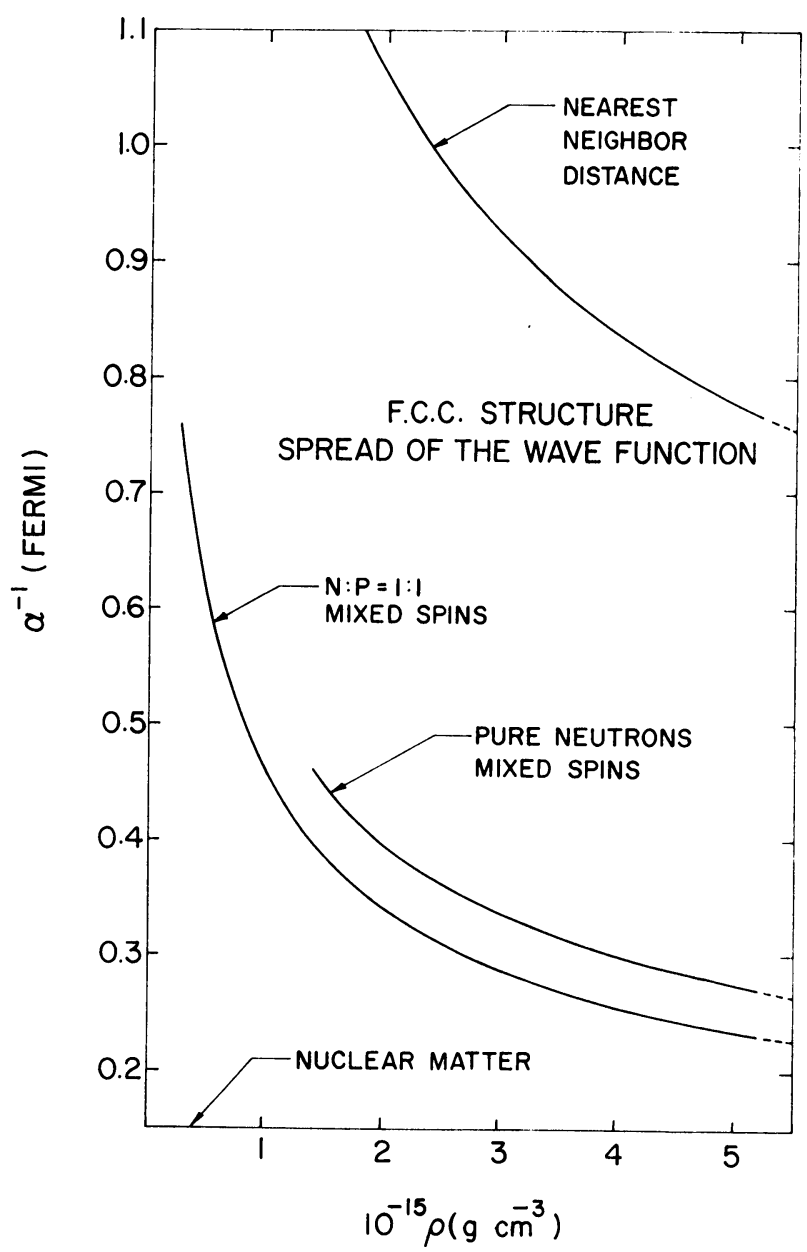

Fig. 10. Spread of the wave function and the nearest neighbor distance as a function of the density.

We have constructed neutron star models composed of cold matter by integrating the equations governing the relativistic stellar structure. For this purpose our equation of state, obtained by assuming a crystalline neutron state, was smoothly joined up with Pandharipande's pure-neutron equation of state at a density of $1.5 \times 10^{15} \mathrm{~g} \mathrm{~cm}^{-3}$. In Figure 12 we show the resulting neutron star masses in units of the solar mass as a 
function of the central density. We find a maximum mass of $1.56 M_{\odot}$ for a stable neutron star at a central density $3 \times 10^{15} \mathrm{~g} \mathrm{~cm}^{-3}$, not very different from a maximum mass of $1.66 M_{\odot}$ calculated by Baym et al. (1971) at a central density of $4.1 \times 10^{15} \mathrm{~g}$ $\mathrm{cm}^{-3}$.

We also attempted a few baryonic crystals by including hyperons in an FCC lattice.

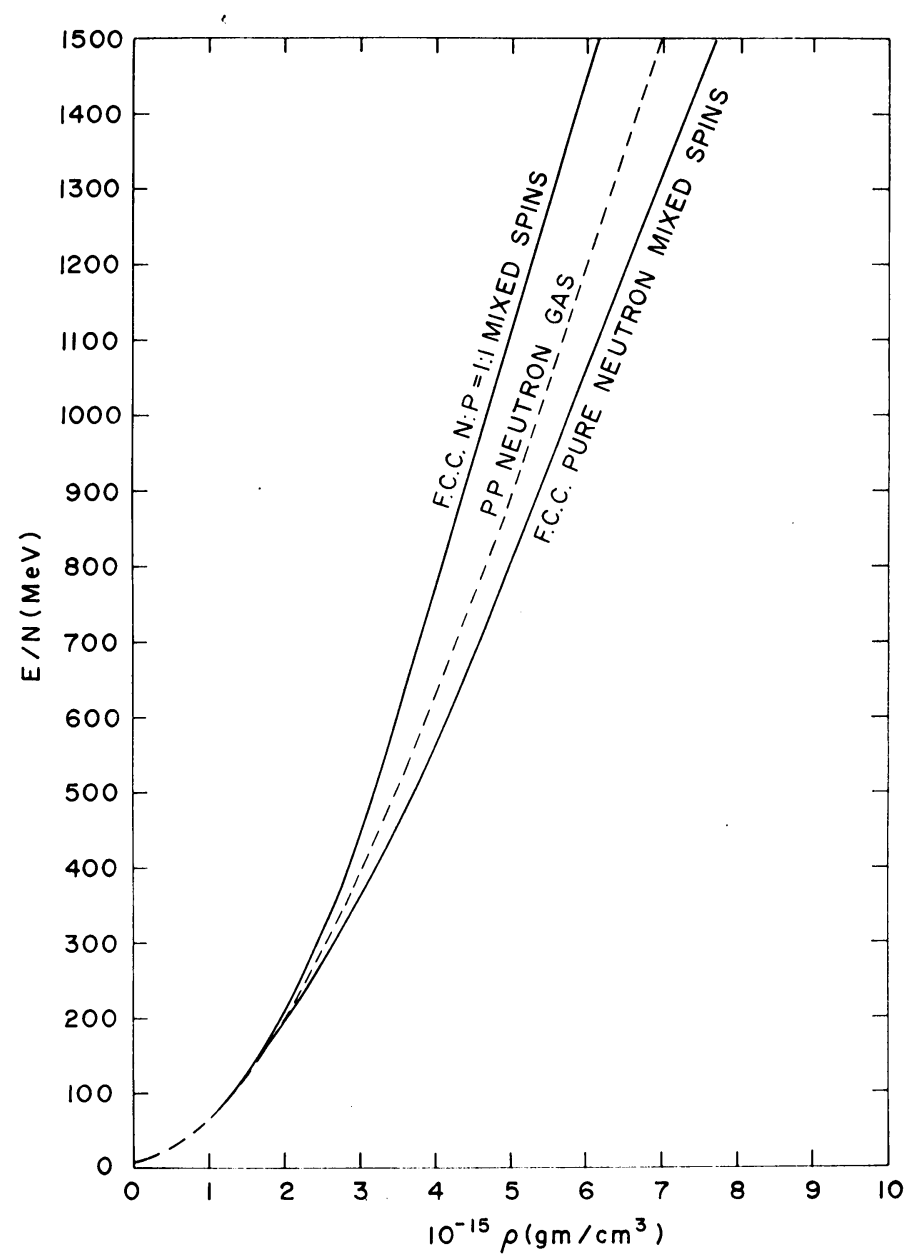

Fig. 11. Energy per particle vs. the density for an FCC lattice made up of pure neutrons and of equal number of neutrons and protons; the dashed line shows Pandharipande's result for neutron gas.

The hyperonic potentials were derived by extending the work of Brown et al. (1970), and a typical hyperonic potential $(\Lambda, N ; T=1 / 2)$ is displayed in Table $\mathrm{V}$. The resulting energies for various distributions of baryons are shown in Figure 13: it is at once evident that the energies are considerably lowered compared to the pure neutron matter arranged in a lattice. One of the principal reasons for this lowering of energy can be 


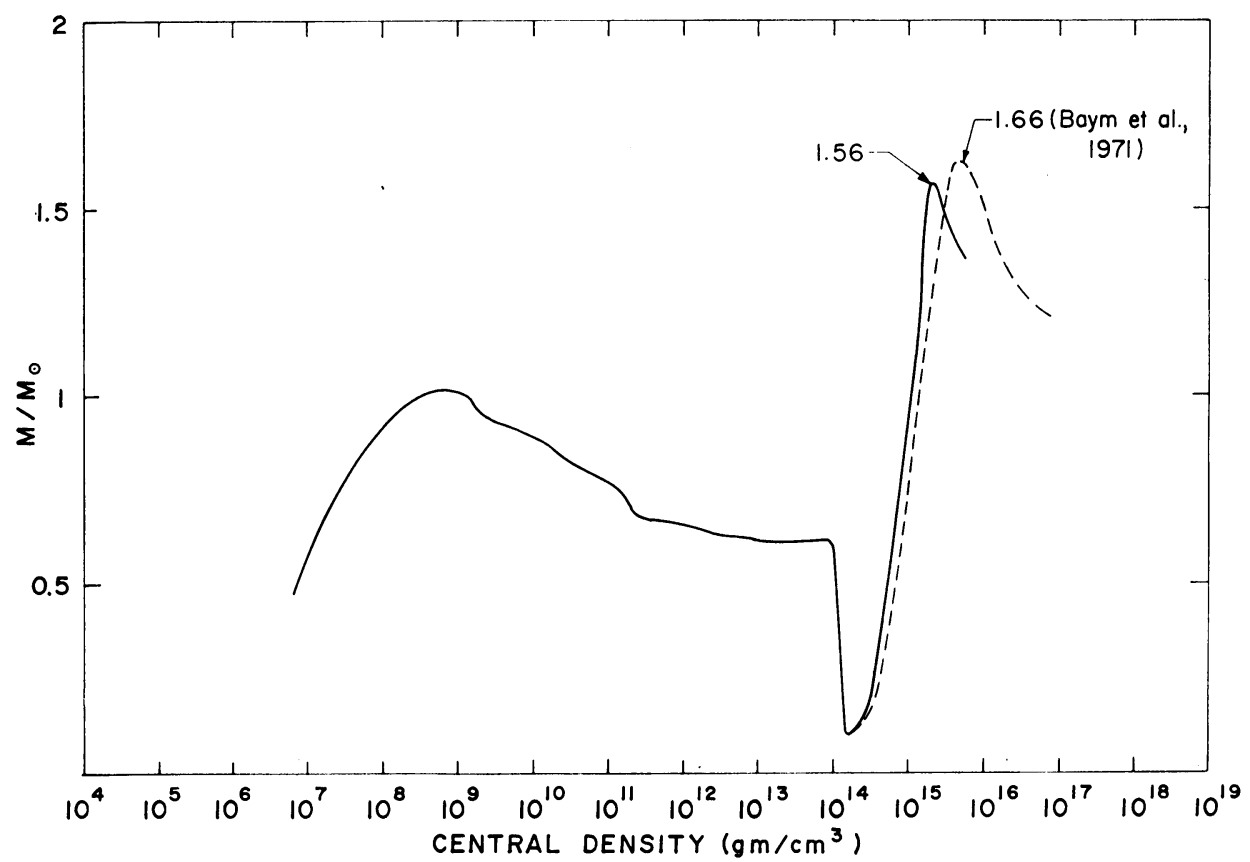

Fig. 12. Neutron star mass in units of the solar mass vs. the central density; the dashed curve shows the results of Baym et al.

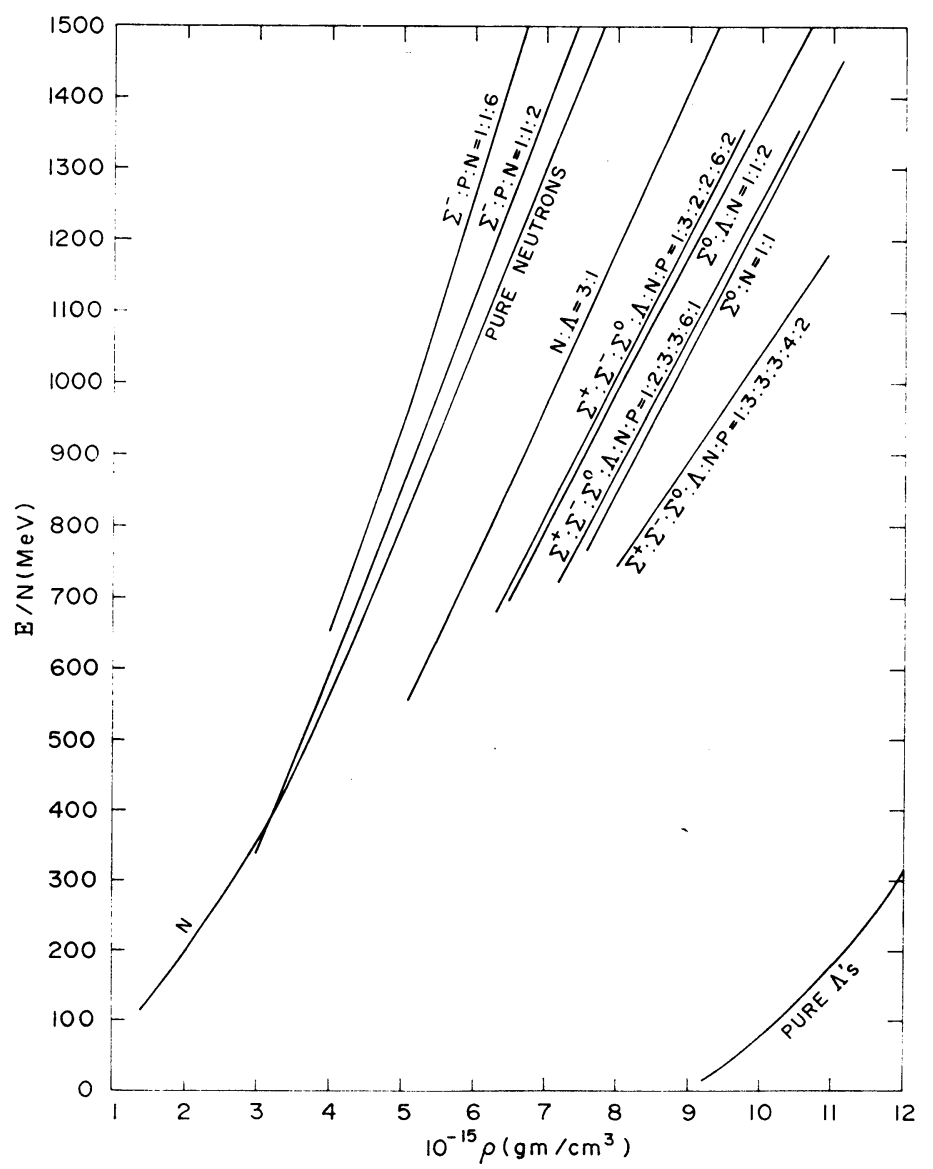


TABLE V

$\Lambda, N$ potential: $T=1 / 2 ; \quad V=V_{\mathrm{C}}+\mathrm{V}_{\sigma} \sigma_{1} \cdot \sigma_{2}+V_{T} S_{12}+V_{\mathrm{LS}} \mathbf{L} \cdot \mathbf{S}+V_{Q} W_{12}$

\begin{tabular}{|c|c|c|c|}
\hline & $\pi(139, T=1$, Ps $)$ & $\eta(548, T=0, \mathrm{Ps})$ & $k(495, T=1 / 2, \mathrm{Ps})$ \\
\hline & $\pi \equiv 0.7 \mathrm{f}^{-1}$ & $\eta \equiv 2.7817 \mathrm{f}^{-1}$ & $k \equiv 2.3465 \mathrm{f}^{-1}$ \\
\hline$V_{\mathrm{C}}$ & 0 & 0 & 0 \\
\hline$V_{\sigma}$ & 0 & $-19.395\left(e^{-\eta r} / r\right)$ & $+(-)^{\mathrm{L}+\mathrm{S} 57.743\left(e^{-k r} / r\right)}$ \\
\hline$V_{T}$ & 0 & $\begin{array}{l}-58.185 f(r)\left(e^{-\eta r} / r\right) \\
f(r)=\frac{1}{3}+0.359 / r+0.129 / r^{2}\end{array}$ & $\begin{array}{c}+(-)^{\mathrm{L}+\mathrm{s}} 173.288 f(r)\left(e^{-k r} / r\right) \\
f(r)=\frac{1}{3}+0.4261 / r+ \\
+0.1816 / r^{2}\end{array}$ \\
\hline$V_{\mathrm{LS}}$ & 0 & 0 & 0 \\
\hline$V_{Q}$ & 0 & 0 & 0 \\
\hline
\end{tabular}

\begin{tabular}{lll}
\hline & $\sigma(490, T=0, \mathrm{~S})$ & $\omega(888, T=0, V)$ \\
\hline & $\sigma \equiv 2.4873 \mathrm{f}^{-1}$ & $\omega \equiv 4.5076 \mathrm{f}^{-1}$ \\
$V_{\mathrm{C}}$ & $-1131.08\left(e^{-\sigma r} / r\right)$ & $+6815.036\left(e^{-\omega r} / r\right)$ \\
$V_{\sigma}$ & $+0.9883 f(r)\left(e^{-\sigma r} / r\right)$ & $+[616.7849-43.6140 f(r)]\left(e^{-\omega r} / r\right)$ \\
& $f(r)=0.402 / r+0.3232 / r^{2}+0.129 / r^{3}$ & $f(r)=0.2218 / r+0.0984 / r^{2}+0.0218 / r^{3}$ \\
& & $-925.177 f(r)\left(e^{-\omega r} / r\right)$ \\
$V_{T}$ & 0 & $f(r)=\frac{1}{3}+0.2218 / r+0.04929 / r^{2}$ \\
& $-135.9047 f(r)\left(e^{-\sigma r} / r\right)$ & $-5754.498 f(r)\left(e^{-\omega r} / r\right)$ \\
$V_{\mathrm{LS}}$ & $f(r)=0.402 / r+0.1616 / r^{2}$ & $f(r)=0.2218 / r+0.04929 / r^{2}$ \\
& $-1.9766 f(r)\left(e^{-\sigma r} / r\right)$ & $+87.228 f(r)\left(e^{-\omega r} / r\right)$ \\
$V_{Q}$ & $f(r)=0.1616 / r^{2}+0.1299 / r^{3}$ & $f(r)=0.0492 / r^{2}+0.0218 / r^{3}$ \\
\hline
\end{tabular}

\begin{tabular}{|c|c|c|}
\hline & $\varrho(755, T=1, V)$ & $k^{*}(890, T=1 / 2, V)$ \\
\hline & $Q \equiv 3.8324 f^{-1}$ & $k^{*} \equiv 4.4275 f^{-1}$ \\
\hline$V_{\mathrm{C}}$ & 0 & $(-)^{\mathrm{L}+\mathrm{S} 552.4166\left(e^{-k^{*}} r / r\right)}$ \\
\hline$V_{\sigma}$ & 0 & $\begin{array}{l}(-)^{\mathrm{L}+\mathrm{S}}[353.103-147.8237 f(r)]\left(e^{-k^{\star} r} / r\right) \\
f(r)=0.2258 / r+0.1020 / r^{2}+0.0230 / r^{3}\end{array}$ \\
\hline$V_{T}$ & 0 & 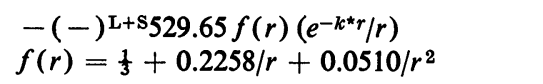 \\
\hline$V_{\text {LS }}$ & 0 & $\begin{array}{l}-(-)^{\mathrm{L}+\mathrm{S}} 1495.686 f(r)\left(e^{-k^{\star} r} / r\right) \\
f(r)=0.2258 / r+0.0510 / r^{2}\end{array}$ \\
\hline$V_{Q}$ & 0 & $\begin{array}{l}(-)^{\mathrm{L}+\mathrm{S}} 295.647 f(r)\left(e^{-k^{\star} r} / r\right) \\
f(r)=0.0510 / r^{2}+0.0230 / r^{3}\end{array}$ \\
\hline
\end{tabular}

attributed to the ${ }^{3} P_{1}$ wave which in the nucleon-nucleon interaction is purely repulsive, but is modestly attractive in all the hyperon-hyperon and hyperon-nucleon interactions. The question of the hyperonic interactions, however, needs to be examined further before any firm conclusions can be drawn. 


\section{Acknowledgement}

It is a pleasure to thank Mal Ruderman and Al Cameron for stimulating discussions.

\section{References}

Anderson, P. W. and Palmer, R. G.: 1971, Nature Phys. Sci. 231, 145.

Banerjee, B., Chitre, S. M., and Garde, V. K.: 1970, Phys. Rev. Letters 25, 1125.

Baym, G., Pethick, C., and Sutherland, P.: 1971, Astrophys. J. 170, 299.

Born, M. and Huang, K.: 1950, Dynamical Theory of Crystal Lattices, Clarendon Press, Oxford. Brandow, B.: 1972, Ann. Phys. 74, 112.

Brown, J. T., Downs, B. W., and Iddings, C. K.: 1970, Ann. Phys. 60, 148.

Clark, J. W. and Chao, N.-C.: 1972, Nature Phys. Sci. 236, 37.

Guyer, R. A.: 1969, in D. Turnbull (ed.), Solid State Phys. 23, 413.

Guyer, R. A. and Zane, I.: 1969, Phys. Rev. 188, 445.

Pandharipande, V. R.: 1971, Nucl. Phys. A174, 641.

Reid, R. V.: 1968, Ann. Phys. 50, 411.

\section{DISCUSSION}

Bethe: When Johnson and I attempted to calculate a crystal model for neutron matter, we found the coupling between higher angular momentum states very important, even though these states have essentially no potential energy. The reason is that the unperturbed wavefunction in relative coordinates,

$$
\phi=\exp -(r-a)^{2} \alpha
$$

is centered at $a$, the lattice distance, and therefore has strong components with large $l$, when expanded around $r=0$. It is legitimate to stop with $l=2$ ?

Canuto: We do not have any reason to believe that the higher waves are unimportant. Since the coupling amongst the waves is very complicated, we have decided to examine results for $l \leqslant 2$. The higher waves will have a strong centrifugal repulsion and as a consequence the wavefunctions for those waves are expected to be pushed away from the origin where the potential is essentially attractive. We therefore feel that if anything their inclusion will lower the energy.

Bethe: Your conclusion is done with essentially the Brueckner-Goldstone method. When I tried to apply this to high density gas, I got much too high binding energy in the 2-body approximation. I realize that many-body clusters are less important for the crystal than for the gas, but still I believe that Pandharipande method is more reliable at high density. In any case, gas and crystal should be done with the same method.

Canuto: I agree that gas and crystal should be computed with the same method. It remains to be seen if a variational method as the one employed by Pandharipande is preferable. Historically speaking, as far as the quantum crystals are concerned, people have gone from variational computation to the Bruckner-Goldstone method and not vice versa. We have employed what quantum crystal physicists have suggested to us as being the best method available.

Bethe: In light of Ruderman's remark after my paper, I withdraw my statement that neutron crystals can not exist at high density. However, I do not believe that Canuto and Chitre have yet proved their existence by the present calculations. 